\title{
Cytogenetics of three Brazilian species of Eleutherodactylus (Anura, Leptodactylidae) with 22 chromosomes and re-analysis of multiple translocations in E. binotatus
}

\author{
Sérgio Siqueira-Jr. ${ }^{1}$, Fernando Ananias ${ }^{2}$ and Shirlei M. Recco-Pimentel ${ }^{1}$ \\ ${ }^{1}$ Universidade Estadual de Campinas, Instituto de Biologia, Departamento de Biologia Celular, \\ Campinas, SP, Brazil. \\ ${ }^{2}$ Universidade São Francisco, Bragança Paulista, SP, Brazil.
}

\begin{abstract}
In this paper, we provide a cytogenetic analysis of Eleutherodactylus guentheri, E. parvus and E. binotatus. All of the species had a diploid chromosomal number of $2 n=22$. The karyotypes of $E$. guentheri and $E$. parvus were very similar and differed only slightly in the morphology of pair 2 . These two species also had an NOR-bearing secondary constriction on the long arms of pair 6 . The karyotype of $E$. binotatus differed from those of $E$. guentheriand $E$. parvus in the morphology and size of the chromosomes, in the number of chromosomal arms, in the NOR location (detected on the short arms of pair 1), and in the pattern of heterochromatin. These results reinforce the differences between $E$. guentheri and $E$. binotatus and support the existence of two species group. Five individuals of $E$. binotatus showed morphs for pairs 2 and 3 . These morphs probably arose from the translocation of a segment from one chromosome of pair 3 to a homologue of pair 2. In addition, some mitotic metaphases of $E$. binotatus showed spontaneous chromosomal breaks which suggested that there were sites of fragility. Meiotic diakinesis showed multiple chromosomal rings, indicating the occurrence of multiple translocations, as previously reported by other investigators. These data suggest that, in addition to fission and fusion, other chromosomal rearrangements were probably involved in the differentiation of the karyotypes of these species of Eleutherodactylus, especially $E$. binotatus.
\end{abstract}

Key words: Eleutherodactylus, heterochromatin, karyotype, nucleolus organizing region (NOR), reciprocal translocation.

Received: December 4, 2003; Accepted: May 25, 2004.

\section{Introduction}

The genus Eleutherodactylus contains nearly 700 species (Frost, 2002), making it the most speciose group of vertebrates. Because of the high diversity among Eleutherodactylus species neither the systematic relationships within the genus nor its detailed taxonomy have been satisfactorily resolved (Kaiser et al., 1995). The genus has been divided into five subgenera (Euhias, Graugastor, Eleutherodactylus, Pelorius and Syrrhopus) (Lynch and Duellman, 1997), with the subgenus Eleutherodactylus consisting of more than 20 species groups. According to Bogart and Hedges (1995), Eleutherodactylus occurs in a wide variety of habitats throughout the Americas, from Argentina to southern North America, with very extensive speciation in the Antilles. Of more than 30 known Brazilian species, 22 occur in southern and southeastern Brazil,

Send correspondence to Shirlei M. Recco-Pimentel. Universidade Estadual de Campinas, Instituto de Biologia, Departamento de Biologia Celular, Caixa Postal 6109, 13083-863 Campinas, SP, Brazil. E-mail: shirlei@unicamp.br. mainly in the Atlantic forest (Castanho and Haddad, 2000). The lack of biological data for South American species of Eleutherodactylus hampers our understanding of the origin of the species in this genus and their phylogenetic relationships to species in other regions.

The heterogeneity and diversity of Eleutherodactylus has led to the proposal of many species groups based only on morphological characters (Garcia, 1996). The species occurring in southern and southeastern Brazil were included in three species groups by Lynch (1976): binotatus, lacteus and parvus. Heyer (1984) proposed an E. guentheri "cluster" with six closely related species, three of which were included in the E. binotatus group by Lynch (1976). All members of the cluster occur in the central and southern regions of the Atlantic forest.

In contrast to their relatively conserved morphology, Eleutherodactylus species show considerable variation in their chromosomal number $(2 \mathrm{n}=18$ to 36$)$ and chromosomal morphology (Bogart, 1973, 1991; Bogart and Hedges, 1995; Kuramoto, 1990). Such variation is uncommon in anurans which, in general, have conserved karyo- 
types within a genus (Bogart, 1973; Morescalchi, 1973). Eleutherodactylus is a rapidly speciating genus and an example of a high rate of chromosomal evolution in frogs (Bogart and Hedges, 1995).

The phylogeny of the South American species of Eleutherodactylus is still poorly known, and studies combining morphological, biochemical, molecular and cytogenetic data are necessary to revise the classification of Eleutherodactylus in order to understand the phylogenetic relationships of this genus better. Whereas several karyological studies have been reported, especially for Central American species of Eleutherodactylus (Bogart, 1970a,b, 1981, 1991; De Weese, 1975; Myamoto, 1983; Schmid et al., 1992; Kaiser, 1995, 1996; Kaiser and Green 1994), little is known about Brazilian species. The karyotypes of E. lacteus (De Lucca and Jim, 1974), E. holti (De Lucca et al., 1974) $(2 \mathrm{n}=20)$, E. guentheri (Beçak, 1968) and E. binotatus (Beçak and Beçak, 1974) $(2 \mathrm{n}=22)$ have been determined by conventional staining.

In this study, we describe the karyotype of E. parvus and re-analyze the chromosomes of E. guentheri and $E$. binotatus. We also provide a more complete cytogenetic analysis of the three species, which represent three different species groups of Eleutherodactylus in southeastern Brazil, and compare the karyotypes of continental populations with those from an ocean island.

\section{Material and Methods}

\section{Animals}

Eleven specimens (eight males and three females) of Eleutherodactylus guentheri, ten specimens (six males and four females) of $E$. parvus and eight specimens (five males and three females) of E. binotatus were collected in the Parque Natural Municipal da Serra do Itapety (PNMSI),

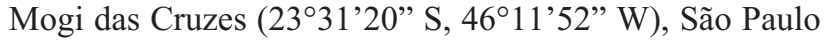
State, Brazil; one female of $E$. parvus and two males of $E$. binotatus were collected at Ubatuba (2326'09" S, $45^{\circ} 04^{\prime} 10^{\prime \prime}$ W), São Paulo State, and two females of $E$. guentheri and three females of $E$. binotatus were collected in the Parque Estadual de Ilha Bela (PEIB) (2346'40" S, $45^{\circ} 21^{\prime} 28^{\prime \prime}$ W), São Paulo State. The specimens were collected in 2001 and 2002, with authorization from the Instituto Brasileiro do Meio Ambiente e Recursos Renováveis (IBAMA - Proc. 02001.008866/01-20). All of the animals were deposited in the "Prof. Adão José Cardoso" Natural History Museum (ZUEC), at the Universidade Estadual de Campinas (UNICAMP) in Campinas, São Paulo State, under the accession numbers 12159-12189 (E. guentheri), (12105-12129) (E. binotatus) and 12130-12158 (E. parvus).

\section{Chromosome preparation and techniques}

Mitotic chromosomes were obtained from intestinal epithelium, testis and bone marrow cell suspensions, as de- scribed by Schmid (1978) and Schmid et al. (1979), after treatment with colchicine for about $3 \mathrm{~h}$. Conventional staining with 10\% Giemsa solution, C-banding (Sumner, 1972), Ag-NOR staining (Howell and Black, 1980) and fluorescence in situ hybridization (FISH) (Viegas-Péquignot, 1992) were used to analyze the chromosomes. The rDNA probe used for FISH consisted of a recombinant plasmid HM123 containing a fragment of Xenopus laevis rDNA (Meunier-Rotival et al., 1979) that was biotin-labeled by a nick translation reaction according to the manufacturer's protocol. The slides were examined with a BX60 Olympus microscope and some of the photographs were obtained using the software Image-Pro Plus, Version 4. The chromosomes were classified according to Green and Sessions (1991).

\section{Results}

\section{Karyotype description}

All individuals of the three species studied had a diploid number of 22 chromosomes. The karyotype of $E$. guentheri consisted of seven pairs of metacentric chromosomes $(1,5,6,8,9,10$ and 11), two pairs of submetacentrics ( 2 and 3 ) and two pairs of subtelocentrics (4 and 7). In some metaphases, secondary constrictions were observed on the long arms of pairs 2, 3, 6 and 8 (all adjacent to the centromere) and on the short arms of pairs 5 and 9 (Figure 5a). In ten specimens, there was size heteromorphism in the secondary constriction between the homologues of pair 6 (Figures 1a and 5a, Table 1).

The karyotype of $E$. parvus was very similar to that of E. guentheri, differing in the morphology of pair 2, which was metacentric in the former species. Secondary constrictions were found on the short arms of pair 2 and on both arms of pair 6 and were heteromorphic between the long arms of the homologues of pair 6 (Figures 1c and 5b, Table 1).

Eleutherodactylus binotatus had a morphologically distinct karyotype in which the chromosomes were 3-4 times bigger than those of the other two species. The karyotype of $E$. binotatus showed $2 \mathrm{n}=22$ chromosomes which consisted of two pairs of metacentrics ( 4 and 7 ), four pairs of submetacentrics (1, 2,6 and 9), two pairs of subtelocentrics ( 3 and 5$)$ and three pairs of telocentrics ( 8 , 10 and 11). In five specimens - two from the continent (one male and one female) and three from Ilha Bela island (two males and one female) - pairs 2 and 3 showed size heteromorphism in the relative length between homologues and differed strongly in their relative size and arm ratios. One of the homologues of pair 2 was metacentric because of the considerable increase in the size of its short arm, and one of the homologues of pair 3 was submetacentric because of the reduction in the size of the long arm (Figures $1 \mathrm{e}, \mathrm{f}$ and $5 \mathrm{c}$, Table 1). A secondary constriction was observed adjacent to the centromere on the short arm of pair 1 


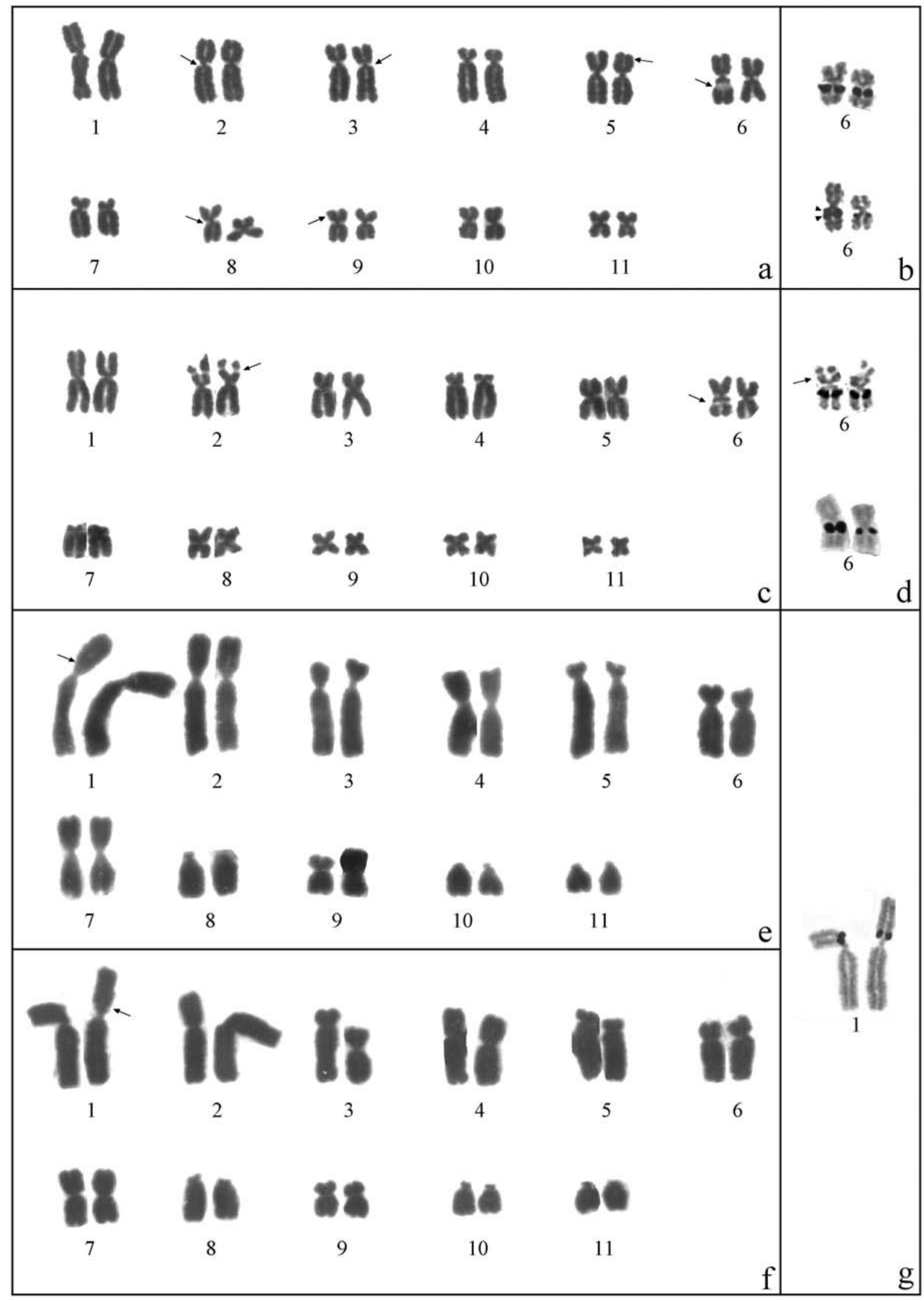

Figure 1 - Karyotypes of E. guentheri (a), E. parvus (c) and E. binotatus (e and $\mathbf{f})$ and NOR-bearing chromosomes of each species (b, d, and $\mathbf{g}$ respectively). The arrows indicate secondary constrictions and the arrowheads in $\mathbf{b}$ indicate the double blocks of NOR. Bar $=10 \mu \mathrm{m}$. 
Table 1 - Morphometric data for mitoic chromosomes of Eleutherodactylus guentheri, E. parvus and E. binotatus. In E. binotatus, the letters a and b indicate the different morphs of chromosomes 2 and 3 found in five specimens.

\begin{tabular}{|c|c|c|c|c|c|c|c|c|c|c|c|c|c|}
\hline & & \multicolumn{12}{|c|}{ Eleutherodactylus guentheri } \\
\hline & N. & 1 & 2 & 3 & 4 & 5 & 6 & 7 & 8 & 9 & 10 & 11 & \\
\hline & RL & 14.25 & 11.92 & 11.04 & 10.23 & 10.10 & 9.23 & 7.49 & 7.25 & 6.50 & 6.32 & 5.68 & \\
\hline & $\mathrm{CR}$ & 1.48 & 1.72 & 1.98 & 3.08 & 1.44 & 1.28 & 3.34 & 1.52 & 1.46 & 1.20 & 1.18 & \\
\hline & $\mathrm{CP}$ & $\mathrm{M}$ & SM & SM & ST & $\mathrm{M}$ & $\mathrm{M}$ & ST & M & M & $\mathrm{M}$ & $\mathrm{M}$ & \\
\hline & & \multicolumn{12}{|c|}{ Eleutherodactylus parvus } \\
\hline & $\mathrm{N}^{\circ}$ & 1 & 2 & 3 & 4 & 5 & 6 & 7 & 8 & 9 & 10 & 11 & \\
\hline & RL & 14.97 & 11.98 & 11.86 & 11.36 & 9.71 & 9.25 & 8.03 & 6.36 & 6.02 & 5.67 & 4.77 & \\
\hline & $\mathrm{CR}$ & 1.34 & 1.44 & 1.85 & 3.90 & 1.27 & 1.09 & 3.92 & 1.16 & 1.21 & 1.40 & 1.16 & \\
\hline & $\mathrm{CP}$ & $\mathrm{M}$ & $\mathrm{M}$ & SM & ST & $\mathrm{M}$ & $\mathrm{M}$ & ST & $\mathrm{M}$ & $\mathrm{M}$ & $\mathrm{M}$ & $\mathrm{M}$ & \\
\hline & & \multicolumn{12}{|c|}{ Eleutherodactylus binotatus } \\
\hline N. & 1 & $2 a$ & $2 b$ & $3 a$ & $3 b$ & 4 & 5 & 6 & 7 & 8 & 9 & 10 & 11 \\
\hline $\mathrm{RL}$ & 15.20 & 13.68 & 16.68 & 11.60 & 8.26 & 10.87 & 9.97 & 9.28 & 8.17 & 6.21 & 5.14 & 5.12 & 4.53 \\
\hline CR & 1.73 & 1.75 & 1.05 & 3.50 & 1.77 & 1.52 & 4.62 & 2.40 & 1.05 & 12.22 & 1.76 & 10.16 & 12.82 \\
\hline CP & SM & SM & $\mathrm{M}$ & $\mathrm{ST}$ & SM & M & $\mathrm{ST}$ & SM & M & $\mathrm{T}$ & SM & $\mathrm{T}$ & $\mathrm{T}$ \\
\hline
\end{tabular}

$\mathrm{RL}=$ relative length $(\%), \mathrm{CR}=$ centromeric ratio, $\mathrm{CP}=$ centromeric position, $\mathrm{M}=$ metacentric, $\mathrm{SM}=$ submetacentric, $\mathrm{ST}=$ subtelocentric, $\mathrm{T}=$ telocentric. Morphometric data were based on measurements of 20 metaphases from 13 specimens of E. guentheri, 18 metaphases from 11 specimens of E. parvus and 19 metaphases from 13 especimens of E. binotatus.

(Figures 1e,g and 5c). Some mitotic metaphases of $E$. binotatus showed spontaneous chromosome breaks, especially in the first six larger chromosomes of the complement (Figure 4e).

\section{Nucleolus organizer region (NOR)}

In E. guentheri and E. parvus, the NORs were present on the long arms of pair 6 , adjacent to the centromeric region and coincident with a secondary constriction observed in Giemsa-stained karyotypes. The NORs were heteromorphic in nine of the 13 specimens $(70 \%)$ of E. guentheri and in eight of the 11 specimens $(73 \%)$ of E. parvus (Figures $1 \mathrm{~b}, \mathrm{~d}$ and $5 \mathrm{a}, \mathrm{b})$. FISH in five specimens of $E$. guentheri and four of $E$. parvus revealed the same regions detected by the silver staining method (Figure 2a,b).

In $E$. binotatus, the NORs were located adjacent to the centromere on the short arms of pair 1, and were detected by silver staining and fluorescence in situ hybridization (Figures $1 \mathrm{~g}$ and $5 \mathrm{c}$ ). One of the specimens with a karyotype containing morphs of chromosomes 2 and 3 had an additional fluorescent label in the telomere of the long arm of subtelocentric pair 3, that was not seen with silver staining (Figure 2c).

\section{C-Banding}

Blocks of strongly stained heterochromatin were located in the centromeric region of all chromosomes of $E$. guentheri and E. parvus and in a few interstitial regions, whereas E. binotatus showed a smaller amount of centromeric heterochromatin. In E. guentheri, small blocks of heterochromatin were also observed adjacent to the centromere on the long arms of pairs 2, 3 and 8 coincident with secondary constrictions. In E. parvus, interstitial bands were detected on the short arms of pairs 2 and 6, coincident with secondary constrictions (Figures $3 \mathrm{a}, \mathrm{b}$ and 5a,b). Eleutherodactylus binotatus showed bands adjacent to the centromere on the short arms of pair 7, on the long arms of pairs 7, 8, 10 and 11, and interstitially on the long arms of pairs 7, 10 and 11 (Figures $3 \mathrm{c}$ and 5c).

\section{Meiosis}

Multiple rings were observed in diakinesis of all male specimens of E. binotatus (Figure 4a-c). Most rings showed three or four pairs of large chromosomes involved in these multiple associations (Figure 4a-c). The same individual of $E$. binotatus always had the same number of chromosomes in all multivalent rings. In individuals with a karyotype containing morphs of chromosomes 2 and 3, the proportion of cells with only bivalents versus cells with multiple rings was $1: 15$, the reverse of normal karyotypes. In some cases, there were differences in the size of homologous bivalents in pairs 2 and 3 (Figure 4d). In E. guentheri and E. parvus, only bivalents were observed.

\section{Discussion}

The diploid number of 22 chromosomes observed in E. guentheri, E. parvus and E. binotatus is common among species of the Leptodactylidae (Kuramoto, 1990), and has been described in about 20 species of Eleutherodactylus. The species analyzed here did not show the large variation in chromosomal numbers reported for some species of Eleutherodactylus (De Lucca et al., 1974; De Lucca and 


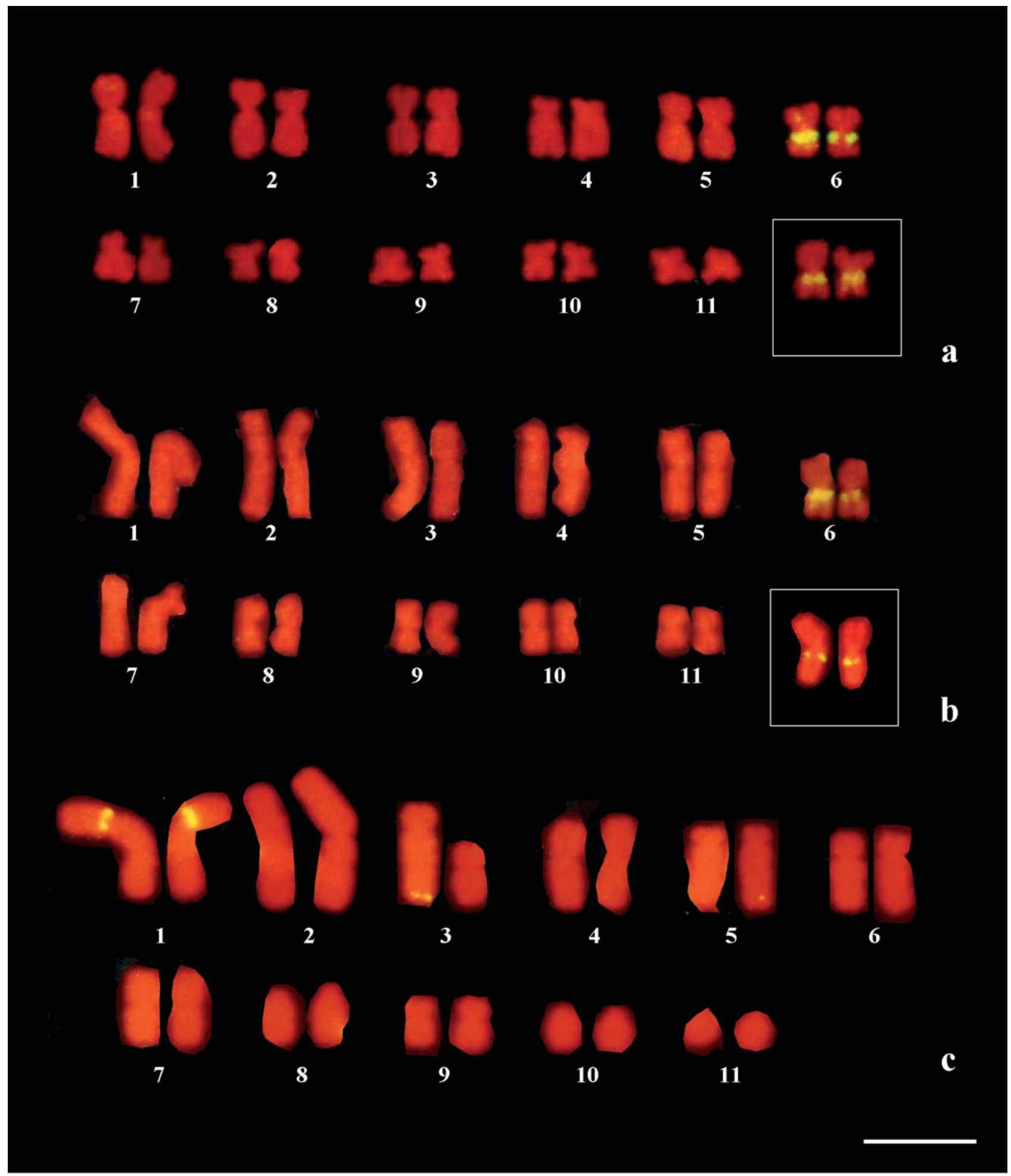

Figure 2 - Metaphases of E. guentheri (a), E. parvus (b) and E. binotatus (c) after fluorescence in situ hybridization with an rDNA probe. Note the additional labeling in one of the homologues of pair 3 in E. binotatus. Bar $=10 \mu \mathrm{m}$.

Jim, 1974; Bogart and Hedges, 1995; Kaiser, 1995). As stated by Bogart (1991), although centric fusions and fissions are the most likely mechanism for changes in chromosomal number in Eleutherodactylus, they are not the only mechanism. There are instances when identical chromosomal numbers have been derived independently (Bogart, 1991). Other mutational events, such as pericentric inversions, translocations, insertions, and dele- 


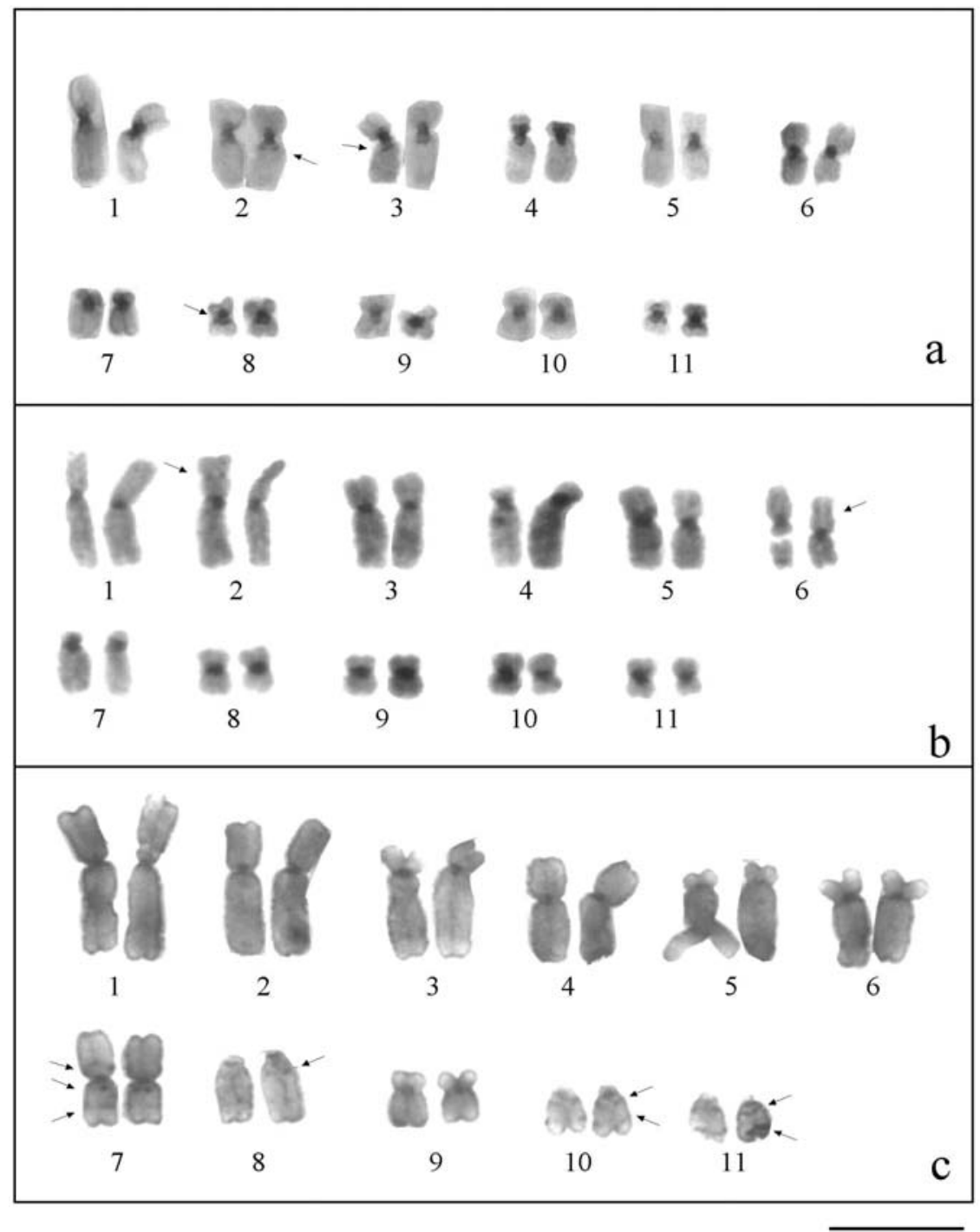

Figure 3 - Karyotypes of E. guentheri (a), E. parvus (b) and E. binotatus (c) after C-banding. The arrows indicate the interstitial heterochromatin. Bar $=10 \mu \mathrm{m}$.

tions could contribute to the chromosomal variation in Eleutherodactylus. Additionally, if centric fusions and fissions are equally likely, then the same number of chromosomes could easily be derived by the convergence of separate lineages (Bogart and Hedges, 1995).

The karyotypes of E. guentheri and E. binotatus differed from those described by Beçak (1968) for specimens of a population from Campos do Jordão, São Paulo State, Brazil, particularly in the classification of some chromosomal pairs and in the number and location of secondary constrictions. Such divergence in chromosome classification may be a technical artifact that reflects the different methods of classification adopted in these karyotypic descriptions. If the classification of Green and Sessions (1991) is applied to the karyotype of E. guentheri described by Beçak (1968), then pairs 5 and 7 are subtelocentrics and pair 9 is metacentric. The same argument applies to pairs 3 and 5 of E. binotatus. Since pairs 4 and 5 of E. guentheri have almost the same size, if their position in the karyogram is reversed then the karyotypes become identical. The differences in the number and locations of the secondary constrictions in E. guentheri and E. parvus may reflect interpopulational chromosomal variation or could be related to the high degree of chromosomal condensation, which would hamper visualization of the constrictions.

We also compared continental specimens of $E$. guentheri and $E$. binotatus with those from an island (Ilha Bela) located about $1.76 \mathrm{~km}$ off the coast of São Paulo State. Ilha Bela is a continental island that originated approximately 11,000 years ago (Vanzolini, 1973). A sea water barrier is particularly interesting since the permeability of amphibian skin makes these animals sensitive to salty water, thereby limiting their ability to travel between islands (Kaiser, 1995) and to the continent. A karyotypic 


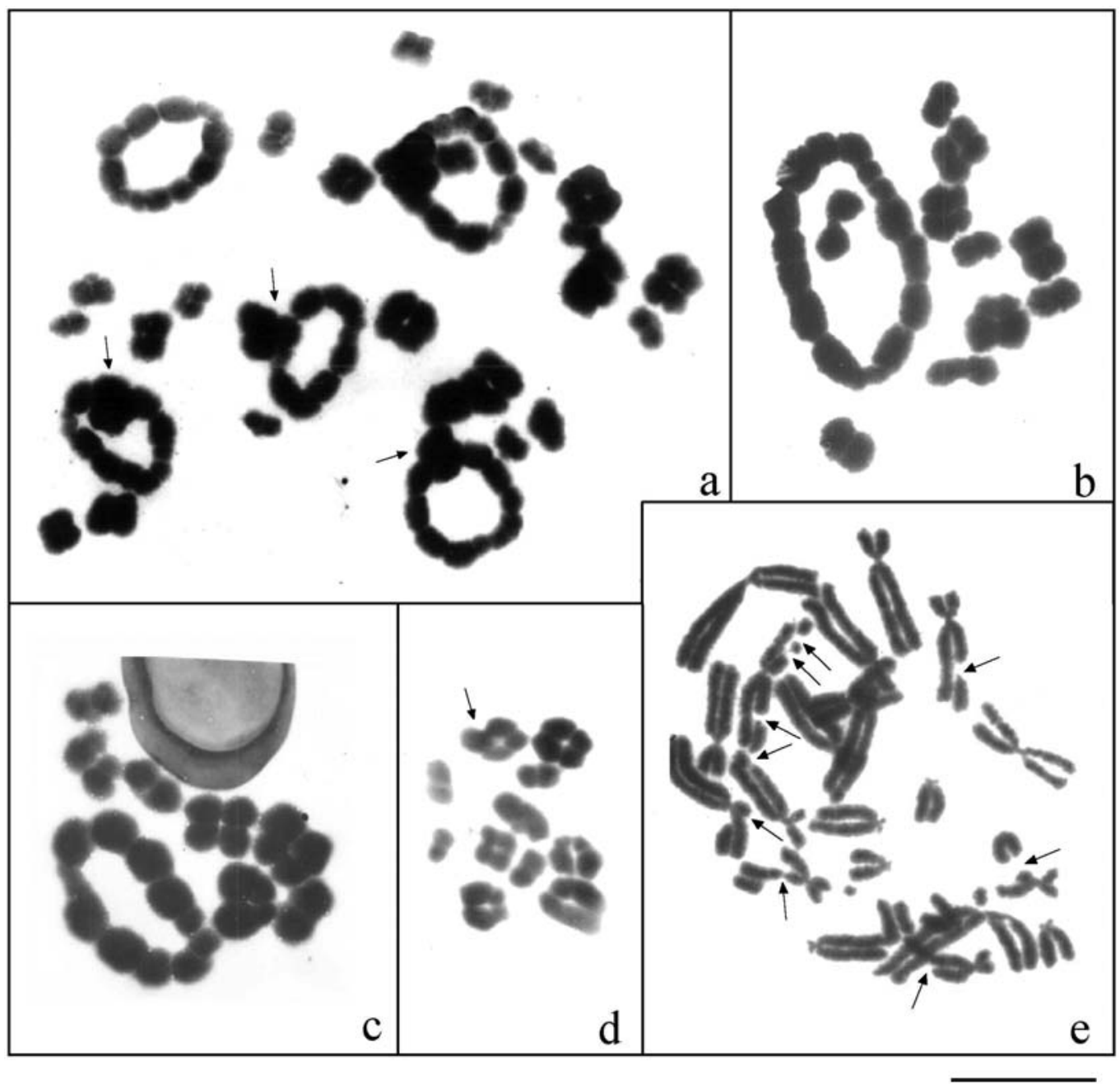

Figure 4 - Diakinesis (a - d) showing multivalent rings in male specimens of E. binotatus. In a and $\mathbf{c}$, the rings have three associated chromosomal pairs, whereas in $\mathbf{b}$, the ring has four associated pairs. The arrows indicate a bivalent that is laterally associated with the multivalent rings. In $\mathbf{d}, 11$ bivalents and their association are shown. The arrow in $\mathbf{d}$ indicates the difference in size between the homologues of pair 3 . In e, the mitotic metaphase of E. binotatus shows spontaneous chromosomal breaks (arrows). Bar $=10 \mu \mathrm{m}$.

analysis of populations separated by a geographic barrier can contribute to studies of speciation and chromosomal evolution. Hass and Hedges (1991) and Gascon et al. (1996, 1998) used morphology and allozymes to study groups of amphibians separated by a river and found no differences between the populations. A river barrier can diminish, but may not completely hinder, gene flow. Our hypothesis that the sea barrier would effectively lead to cytogenetic divergences was not confirmed since no karyotypic variation was observed between mainland and island populations of E. guentheri and E. binotatus. However, further populational analyses are necessary since one specimen of E. binotatus from Queimada Grande island did not show multiple translocations (Beçak and Beçak, 1974).

Of all the secondary constrictions, only that on the long arms of pair 6 was located at the same position and showed the same heteromorphism in E. guentheri and $E$. parvus. This constriction correspond to the NOR-bearing region of this chromosome pair in both species. In addition to the marked similarity between the chromosomal morphologies of the E. guentheri and E. parvus karyotypes, the same NOR location may also denote a very close relationship between the species, as proposed by Schmid (1982) and Schmid et al. (1990). The NOR heteromorphism seen in some individuals of both species apparently involved a tandem duplication of the NOR since two distinct blocks were observed with FISH and silver staining. As stated by Schmid (1982) and Schmid et al. (1990), unequal meiotic crossing-over and sister chromatid exchanges could give rise to heteromorphic NOR, including duplications and deletions.

In one individual of E. guentheri and two of $E$. parvus, only one chromosome of pair 6 was silver-stained. Similar cases have been described for Xenopus laevis (Elsdale et al., 1958) and Bufo fowleri (Schmid, 1982), and may have resulted from a deletion, although in these cases 


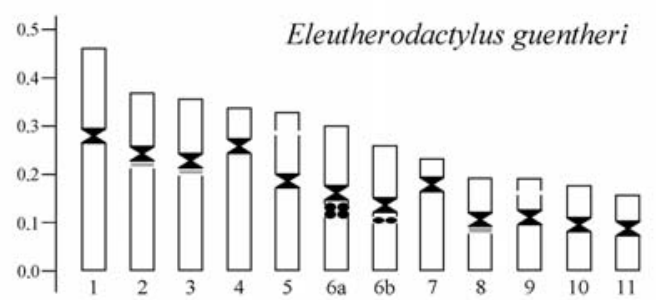

a

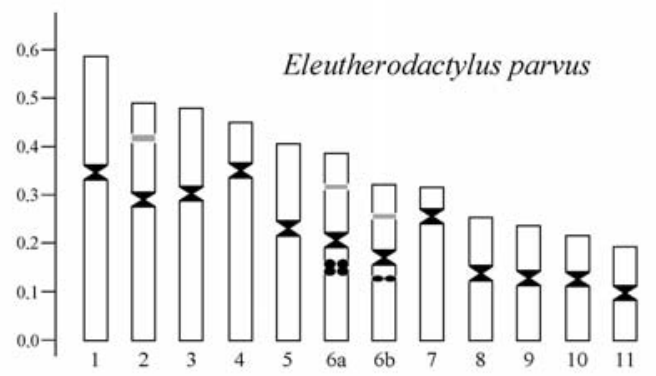

b

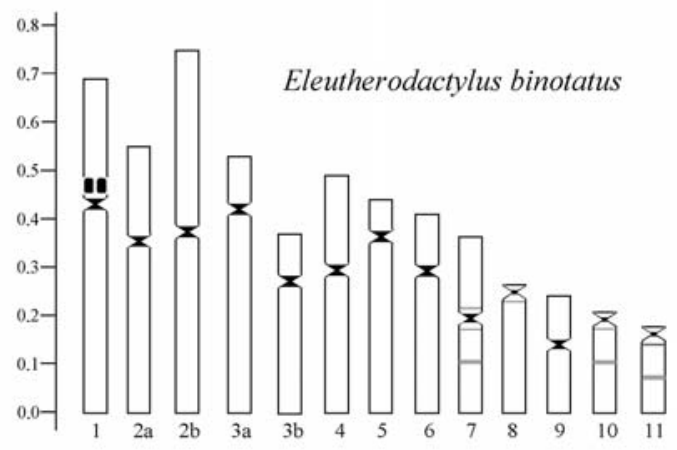

Figure 5 - Ideograms of the karyotypes of E. guentheri (a), E. parvus (b) and E. binotatus (c). Solid areas: dark C-bands. Gray areas: faint C-bands. Dark circles: NORs. Open regions: secondary constrictions. In $\mathbf{c}$, the letters $\mathbf{a}$ and $\mathbf{b}$ indicate the different morphs of chromosomes 2 and 3 .

the absence of rDNA was not confirmed by molecular techniques. In E. parvus and E. guentheri, both homologues were labeled by FISH, although the labeling was very small in one homologue. This observation suggests that the NOR was not completely deleted and that silver staining was not effective in detecting such a small amount of rDNA, in contrast to FISH.

Blocks of strongly stained heterochromatin were located in the centromeric region of all chromosomes of $E$. guentheri and E. parvus and in a few interstitial regions, whereas E. binotatus showed a smaller amount of centromeric heterochromatin. Bogart (1973) suggested that species with very similar karyotypes had a common ancestry. This may be the case for E. guentheri and E. parvus, which differed only slightly in the morphology of pair 2 and in the pattern of the interstitial heterochromatin. This chro- mosome was submetacentric in E. guentheri and metacentric in E. parvus, with the short arm being bigger in E. parvus, probably because of the presence of a secondary constriction associated with a heterochromatic block. Bogart (1981) and King (1991) suggested that reciprocal translocations, inversions and additions of heterochromatic blocks may have occurred during the recent phylogenesis of this genus.

The great similarity between the karyotypes of $E$. guentheri and E. parvus may be indicative of their close phylogenetic relationship. Likewise, Kaiser et al. (1995) reported that the karyotypes of E. charlottevilensis and $E$. terraebolivianus also showed great similarity at the morphological levels and concluded that these species were closely related.

Despite having the same number of chromosomes $(2 \mathrm{n}=22)$, the karyotype of $E$. binotatus differed from those of E. guentheri and E. parvus in the number of chromosomal arms (38 in E. binotatus and 44 in E. guentheri and E. parvus), in the larger size of its chromosomes [Beçak and Beçak (1974) reported that the genome of E. binotatus was four times greater than in E. guentheri], in the presence of telocentric chromosomes, in the NOR-bearing chromosomes, and in the amount and distribution of heterochromatin. Together, these differences indicate that distinct chromosomal rearrangements occurred during the evolution of these taxa.

The presence of multivalent rings during meiosis in E. binotatus indicated multiple translocations, as previously suggested by Beçak and Beçak (1974). The variable relative size and the arm ratios of the different morphs of chromosomes 2 and 3 in E. binotatus may have been generated by heterozygous translocation. This hypothesis is supported by the presence of multiple translocations and of unequal pairing between the homologues of pairs 2 and 3 during diakinesis in males. No individuals had both homologues of the translocated morphs of pairs 2 and 3, perhaps because this was a lethal combination that may have led to developmental disturbances in early embryogenesis. The chromosomal breaks seen in some metaphases also indicated that there were fragile sites in the karyotype of $E$. binotatus that allowed chromosomal rearrangements.

Apparently, only pairs 1 to 6 could be involved in the formation of the big multivalent ring, with pair 8 or 9 being the bivalent associated laterally with the multivalent ring. The lack of association of some chromosomes (pairs 7, 10 and 11 , which remain as bivalents) with the multivalent rings could be related to the presence of interstitial heterochromatic regions in these chromosomes that could prevent pairing between homologous chromosomal arms. This hypothesis was proposed for Physalaemus petersi by Lourenço et al. (2000), who described the second case of a multivalent meiotic configuration in Anura.

The rDNA site detected only by FISH in the telomere of one homologue of pair 3 of E. binotatus could also be ex- 
plained by the translocation of homologous rDNA sequences. The association of the NOR-bearing chromosomal arm of pair 1 with the long arm or pair 3 that carried the additional rDNA site reinforced the hypothesis of translocations as the main mechanism involved in this chromosomal rearrangement. Nevertheless, we cannot exclude the possibility that other mechanisms of dispersion, such as those discussed by Foote et al. (1991), Schmid et al. (1995) and Lourenço et al. (1998, 2000), are also involved, although they are less probable. These mechanisms include the transposition of mobile genetic elements, ribosomal cistron amplification and rDNA reinsertion errors during extra chromosomal amplification of ribosomal cistrons. Cases of additional labels detected in one of the homologues by FISH but not seen by silver-staining have also been described in Hyla chrysoscelis and $H$. versicolor (Wiley et al., 1989), H. nana (Medeiros et al., 2003), and Colostethus sp. aff. marchesianus (Veiga-Menoncello et al., 2003).

The great similarity in the karyotypic morphology and NOR location of E. guentheri and E. parvus compared to the larger DNA content and divergent karyotype (size of the chromosomes and number of chromosomal arms and telocentrics) and NOR location in E. binotatus, indicates a divergence from the latter species. Beçak and Beçak (1974) suggested that polyploidy combined with interstitial duplications was the most probable explanation for the drastic increase in the DNA content of E. binotatus, and that the multivalent meiotic ring observed in this species was caused by translocations that occurred after polyploidy.

Our cytogenetic data do not agree with the placement of E. guentheri in the binotatus group as proposed by Lynch (1976) based on morphological data. The $E$. guentheri cluster suggested by Heyer (1984) is more consistent with our results. The chromosomal information for the three karyotypes examined here reinforces the differences between E. guentheri and E. binotatus and supports the existence of two species group, although a detailed cytogenetic analysis of other species of the three groups (binotatus, guentheri and parvus) and of the E. lacteus group is necessary to support sister-group relationships or wider affinities of these taxa.

The Giemsa-stained karyotypes of E. holti $(2 \mathrm{n}=20)$ (De Lucca et al., 1974) and E. lacteus $(2 \mathrm{n}=20)$ (De Lucca and Jim, 1974) are more similar to E. guentheri and E. parvus than to $E$. binotatus. The divergence in the karyotypes of E. guentheri, E. parvus, E. lacteus and E. holti appears have involved rearrangements in the smaller group of chromosomes since the six largest pairs are morphologically very similar.

Based on the six species analyzed and the proposed groups, southern and southeastern Brazil may have only one group of Eleutherodactylus with a low chromosomal number that is apparently fixed at around 20 to 22 . However, further cytogenetic studies on other Brazilian species are necessary to confirm this hypothesis. Additional studies are also needed to determine whether the large genome size of $E$. binotatus is also present in other species, especially those of the binotatus species group, and to assess whether the karyotypes of other species of the E. guentheri and $E$. parvus groups are closely related.

\section{Acknowledgments}

We thank Marcos M.A. Yamamoto and Débora Cafaro for helping with the field-work, and Klélia Aparecida de Carvalho and Lilian Ricco Medeiros for helping with the FISH experiments. This research was supported by CAPES (Coordenação de Aperfeiçoamento de Pessoal de Nível Superior) and FAPESP (Fundação de Amparo à Pesquisa do Estado de São Paulo, grant no. 00/02461-5 and Programa BIOTA).

\section{References}

Beçak ML (1968) Chromosomal analysis of eighteen species of Anura. Caryologia 21:191-208.

Beçak ML and Beçak W (1974) Diploidization in Eleutherodactylus (Leptodactylidae - Amphibia). Experientia 30:624-625.

Bogart JP (1970a) Los cromosomas de anfibios del genero Eleutherodactylus. Jact IV Congr Latin Zool 1:65-78.

Bogart JP (1970b) Systematic problems in the amphibian family Leptodactylidae (Anura) as indicated by karyotypic analysis. Cytogenetics 9:369-383.

Bogart JP (1973) Evolution of anuran karyotypes. In: Vial JL (ed) Evolutionary Biology of Anurans. University of Missouri Press, Columbia, pp 337-349.

Bogart JP (1981) Chromosome studies in Sminthillus from Cuba and Eleutherodactylus from Cuba and Puerto Rico (Anura: Leptodactylidae). Ont Mus Life Sci Contrib n. 129.

Bogart JP (1991) The influence of life history on karyotypic evolution in frogs. In: Green DM and Sessions SK (eds) Amphibian Cytogenetics and Evolution. Academic Press, New York, pp 233-258.

Bogart JP and Hedges SB (1995) Rapid chromosome evolution in Jamaican frogs of the genus Eleutherodactylus (Leptodactylidae). J Zool 235:9-31.

Castanho LM and Haddad CFB (2000) New species of Eleutherodactylus (Amphibia: Leptodactylidae) from Guaraqueçaba, Atlantic forest of Brazil. Copeia 3:777-781.

De Lucca EJ and Jim J (1974) Cromossomos de alguns Leptodactylidae. Rev Bras Biol 34:407-410.

De Lucca EJ, Jim J and Foresti F (1974) Chromosomal studies in twelve species of Leptodactylidae and one Brachycephalidae. Caryologia 27:183-192.

De Weese JE (1975) Chromosomes in Eleutherodactylus (Anura: Leptodactylidae). Mamm Chrom Newslett 16:121-123.

Elsdale TR, Fischberg M and Smith SA (1958) A mutation that reduces nucleolar number in Xenopus laevis. Exp Cell Res 14:642-643.

Foote DL, Wiley JE, Little DL and Meyene J (1991) Ribosomal RNA gene site polymorphism in Bufo terrestris. Cytogenet Cell Genet 57:196-199. 
Frost DR (2002) Amphibian species of the world. Version 2.21 (15 July 2002). http://research.amnh.org/herpetology/ ampibia/index.htm.

Garcia PCA (1996) Nova espécie de Eleutherodatylus Duméril and Bibron 1981, do Estado de Santa Catarina, Brasil (Amphibia; Anura; Leptodfactylidae). Biociências 2:57-68.

Gascon C, Lougheed SC and Bogart JP (1996) Genetic and morphological variation in Vanzolinius discodactylusi: A test of the river hypothesis of speciation. Biotropica 28:376-387.

Gascon C, Lougheed SC and Bogart JP (1998) Patterns of genetic population differentiation in four species of Amazonian frogs: a test of riverine barrier hypothesis. Biotropica 30:104-119.

Green MG and Sessions SK (1991) Nomenclature for chromosomes. In: Green DM and Sessions SK (eds) Amphibian Cytogenetics and Evolution. Academic Press, San Diego, pp 431-432.

Hass CA and Hedges SB (1991) Albumin evolution in West-Indian frogs of the genus Eleutherodactylus (Leptodactylidae) - Caribbean biogeography and a calibration of the albumin immunological clock. J Zool 225:413-426.

Heyer RW (1984) Variation, Systematics, and Zoogeography of Eleutherodactylus guentheri and Closely Related Species (Amphibia:Anura:Leptodactylidae). Smithsonian Institution Press, Washington, $42 \mathrm{pp}$.

Howell WM and Black DA (1980) Controlled silver-staining of nucleolus organizer regions with a protective colloidal developer: 1-step method. Experientia 36:1014-1015.

Kaiser H (1995) Caribbean frogs: How molecules and genes help to resolve questions of their systematics, evolution and biogeography. Futura 2:88-95.

Kaiser H (1996) Systematics and biogeography of eastern Caribbean Eleutherodactylus (Anura: Leptodactylidae): Consensus from a multidisciplinary approach. Contrib Herpetol 12:129-140.

Kaiser H and Green DM (1994) Systematics and biogeography of eastern Caribbean frogs (Leptodactylidae: Eleutherodactylus), with the description of a new species from Dominica. Can J Zool 72:2217-2237.

Kaiser H, Dwyer CM, Feichtinger W and Schmid M (1995) A new species of Eleutherodactylus (Anura: Leptodactylidae) from Tobago, West Indies and its morphometric and cytogenetic characterization. Herpet Nat Hist 3:151-163.

King M (1991) The evolution of heterochromatin in the Amphibia genome. In: Green DG and Sessions SK (eds) Amphibian Cytogenetic and Evolution. Academic Press, San Diego, pp 359-391.

Kuramoto M (1990) A list of chromosome numbers of anuran amphibians. Bull Fukuoka Univ Educ 39:83-127.

Lourenço LB, Recco-Pimentel SM and Cardoso AJ (1998) Polymorphism of the nucleolus organizer regions (NORs) in Physalaemus petersi (Amphibia, Anura, Leptodactylidae) detected by silver staining and fluorescence in situ hybridization. Chrom Res 6:621-628.

Lourenço LB, Recco-Pimentel SM and Cardoso AJ (2000) A second case of multivalent meiotic configurations in diploid species of Anura. Genet Mol Biol 23:131-133.

Lynch JD (1976) The species groups of the South American frogs of the genus Eleutherodactylus (Leptodactilydae). Occ Pap Mus Nat Hist Univ Kans 61:1-24.
Lynch JD and Duellman WE (1997) Frogs of the genus Eleutherodactylus in western Ecuador: systematics, ecology and biogeography. Mus Nat Hist Univ Kans Spec Publ 23:1-236

Medeiros LR, Rossa-Ferres DC and Recco-Pimentel SM (2003) Chromosomal differentiation of Hyla nana and Hyla sanborni (Anura, Hylidae) with a description of NOR polymorphism in H. nana. J. Hered 94:149-154.

Meunier-Rotival M, Cortadas J, Macaya G and Bernardi G (1979) Isolation and organization of calf ribosomal DNA. Nucleic Acids Res 6:2109-2123.

Miyamoto MM (1983) Frogs of the Eleutherodactylus rugulosus group: A cladistic study of allozyme, morphological, and karyological data. Syst Zool 32:109-124.

Morescalchi A (1973) Amphibia. In: Chiarelli AB and Capanna G (eds) Cytotaxonomy and Vertebrate Evolution. Academic Press, London, pp 233-248.

Schmid M (1978) Chromosome banding in Amphibia I. Constitutive heterochromatin and nucleolus organizer regions in Bufo and Hyla. Chromosoma 66:361-388.

Schmid M (1982) Chromosome banding in Amphibia VII. Analysis of the structure and variability of NORs in Anura. Chromosoma 87:327-344.

Schmid M, Feichtinger W, Wimer R, Mais C, Bolaños F and Leon $\mathrm{P}$ (1995) Chromosome banding in Amphibia XXI. Inversion polymorphism and nucleolus organizer regions in Agalychnis callidryas (Anura, Leptodactylidae). Chromosoma 101:284-292.

Schmid M, Olert J and Klett C (1979) Chromosome banding in Amphibia. III. Sex chromosomes in Triturus. Chromosoma 71:29-55.

Schmid M, Steinlein C and Feichtinger W (1992) Chromosome banding in Amphibia XVII. First demonstration of multiple sex chromosomes in amphibian: Eleutherodactylus maussi (Anura, Leptodactylidae). Chromosoma 101:284-292.

Schmid M, Steinlein C, Frield R, De Almeida CG, Haaf T, Hillis DM and Duellman WE (1990) Chromosome banding in Amphibia XV. Two types of $\mathrm{Y}$ chromosome and heterochromatin hypervariability in Gastrotheca pseustes (Anura, Hylidae). Chromosoma 99:413-423.

Sumner AT (1972) A simple technique for demonstrating centromeric heterochromatin. Exp Cell Res 75:304-306.

Vanzolini PE (1973) Distribution and differentiation of animals along the coast and on continental islands of the state of São Paulo, Brazil. 1. Introduction to the area and problems. Pap Avul Zool 26:281-294.

Veiga-Menoncello AC, Lima AP and Recco-Pimentel SM (2003) Cytogenetic analysis of four Central Amazonian species of Colostethus (Anura, Dendrobatidae) with a diploid complement of 22 chromosomes. Hereditas 139:189-198.

Viegas-Péquignot E (1992) In situ hybridization to chromosomes with biotinylated probes. In: Willernson D (ed) In Situ Hybridization: A Practical Approach. Oxford University PressIRL Press, Oxford, pp 137-158.

Wiley JE, Little ML, Romano MA, Blount DA, and Cline GR (1989) Polymorphism in the location of the $18 \mathrm{~S}$ and $28 \mathrm{~S}$ rDNA genes on the chromosomes of the diploid-tetraploid treefrogs Hyla chrysoscelis and H. versicolor. Chromosoma 97:481-487.

Associate Editor: Yatiyo Yonenaga-Yassuda 